\title{
SLEEP DISTURBANCE IN SCLERODERMA
}

Kamilla de Bessa Jorge ${ }^{1, \star}$, Laís Zanlorenzi ${ }^{1}$, Anauá Fernanda dos Santos Cavalcante ${ }^{1}$, Natália de Oliveira ${ }^{1}$, Nícolas Silva ${ }^{1}$, Renato Nisihara ${ }^{1}$, Patrícia Martin ${ }^{1}$, Thelma Larocca Skare ${ }^{1}$

1.Faculdade Evangélica Mackenzie, Curitiba (PR), Brazil.

*Corresponding author: kamillabessajorge@gmail.com

\section{BACKGROUND}

Sleep quality may be impaired in scleroderma (SSc) patients, impacting on quality of life. Hence, we evaluated the prevalence of sleep disturbance in SSc patients and its possible association with epidemiological, clinical and laboratory data, the approach to treatment, depression and its impact on quality of life.

\section{MATERIALS AND METHODS}

This is a cross sectional study including 60 SSc patients. Epidemiological, clinical, laboratory and treatment data were extracted from the charts. The Pittsburg questionnaire (PSQI) was used to evaluate sleep quality, the Center for Epidemiological Scale Depression (CES-D) to evaluate depression, and the 12-Item Short-Form Health Survey (SF-12) for quality of life. Disease severity was evaluated by the Medsger index and the degree of cutaneous involvement by the Rodnan modified (Rodnan-m).

\section{RESULTS}

The prevalence of patients with sleep disturbance was of 73.3\%. Sleep disturbance was associated with esophageal involvement $(p=0.03)$, Medsger index ( $p=0.01)$, and depression $(p=0.002)$. Patients with poor quality of sleep had worse quality of life by the SF-12 in mental ( $p=0.001)$ and physical domains $(p=0.0008)$. No associations were found with epidemiological, serological and treatment variables (all $p=n s$ ).

\section{CONCLUSIONS}

There is a high prevalence of sleep disturbance in SSc that is associated with esophageal involvement, severity of disease, depression and quality of life. 